\title{
Distribuição longitudinal de fertilizante granulado em diferentes inclinações e posição da rosca de um dosador de rosca helicoidal dupla
}

\section{Longitudinal distribution of granulated fertilizer in different tiltings and position of the thread position of a double helicoidal doser}

\author{
Júnior Verardi', David Peres da Rosa ${ }^{2}$, Artur Zancan ${ }^{3}$, Paulo Conte ${ }^{4}$, \\ Marcos Longaretti ${ }^{5}$, Roger Toscan Spagnolo ${ }^{6}$
}

Verardi, J; Peres da Rosa, D; Zancan, A; Conte, P; Longaretti, M; Toscan-Spagnolo, R. Distribuição longitudinal de fertilizante granulado em diferentes inclinações e posição da rosca de um dosador de rosca helicoidal dupla. Tecnología en Marcha. Vol. 32, Especial. XIII CLIA. Abril 2019.

Pág 128-134.

DOI: https://doi.org/10.18845/tm.v32i7.4272

1 Acadêmico do curso bacharel em Agronomia do Instituto Federal de Educação Ciência e Tecnologia do Rio Grande do Sul (IFRS) - Campus Sertão (RS) bolsista BICTES/IFRS, Núcleo de Estudos em Solos e Máquinas Agrícolas (NESMA). Brasil. E-mail: junior.verardi@hotmail.com

2 Eng. Agríc., Prof. Dr do IFRS - Campus Sertão, Sertão - RS, NESMA. Brasil. E-mail: david.darosa@sertao.ifrs.edu.br

3 Acadêmico do curso Bacharel em Agronomia do IFRS - Campus Sertão, Sertão - RS, NESMA. Brasil. E-mail: zancan.artur@gmail.com

4 Acadêmico do curso Bacharel em Agronomia do IFRS - Campus Sertão, Sertão - RS, NESMA. Brasil. E-mail: pauloconte20@gmail.com

5 Acadêmico do curso Bacharel em Agronomia do IFRS - Campus Sertão, Sertão - RS, NESMA. Brasil. E-mail: marcos.longaretti@hotmail.com

6 Eng. Agríc., Prof. Dr da UFPel, Pelotas - RS. Brasil. E-mail: roger.toscan@gmail.com 


\section{Palavras chave}

Rosca dupla; coeficiente de variação; teste de bancada

\section{Resumo}

A distribuição de fertilizante por dosadores de rosca helicoidal é um fator essencial para o estabelecimento e rendimento das culturas. O objetivo do trabalho foi mensurar a eficiência de um novo dosador de fertilizante de rosca dupla. O experimento foi realizado em bancada de teste com uma esteira $\left(5.4 \mathrm{~km} \mathrm{~h}^{-1}\right)$, empregando delineamento inteiramente casualisado. Foi usado dose fixa de $220 \mathrm{~kg} \mathrm{ha}^{-1}$, sendo que foram testadas 3 roscas $(22 \mathrm{~mm}, 17 \mathrm{~mm}$, e $34 \mathrm{~mm}$ ) trabalhando a $54 \mathrm{rpm}$. Cada teste era realizada coletas a cada $0.09 \mathrm{~m}$ em um total de $5.4 \mathrm{~m}$, sendo simulado 3 inclinações longitudinais: $0^{\circ} ;-11^{\circ} \mathrm{e}+11^{\circ}$, e 4 posições de acoplamento da rosca no equipamento, posição $A$ - as duas roscas na mesma posição, B - rosca da direita posicionada para cima, C - roscas totalmente contrarias e D - rosca da direita para baixo, com 3 repetições. Como parâmetro avaliador foram: coeficiente de variação (CV) distribuição do fertilizante em relação a inclinação de trabalho e posição das roscas e amplitude de distribuição. Não houve diferença significativa entre as inclinações testadas, em nível o CV médio foi de $36.29 \%$, em $-11^{\circ}$ foi de $37,11 \%$ e $+11^{\circ}$ foi $36.57 \%$. Diferenças ocorreram nas diferentes posições de trabalho e tipo de roscas, sendo CV na posição A de 38.65\%, B 37.18\%, C 35.63\% e D 35.18\%. O dosador, apresenta uma variação da distribuição relativamente alta, quanto menor o passo da rosca menor é a variação da distribuição, sendo que a posição de encaixe da rosca não altera significativamente desempenho.

\section{Keywords}

Double thread; coefficient of variation; bench test.

\section{Abstract}

The distribution of fertilizer by helical screw feeders is an essential factor for the establishment and yield of the crops. The objective of this work was to measure the efficiency of a new fertilizer of double thread fertilizer. The experiment was carried out on test bench with a treadmill (5.4 $\mathrm{km} \mathrm{h}^{-1}$ ), using a completely randomized design, using a test bench equipped with a treadmill. A fixed dose of $220 \mathrm{~kg} \mathrm{ha}^{-1}$ was used, and 3 threads $(22 \mathrm{~mm}, 17 \mathrm{~mm}$, and $34 \mathrm{~mm})$ were tested at 54 rpm. Each test was performed every $0.09 \mathrm{~m}$ in a total of $5.4 \mathrm{~m}$, being simulated 3 longitudinal slopes: $0^{\circ} ;-11^{\circ}$ and $+11^{\circ}$, and 4 thread coupling positions in the machine, position $\mathrm{A}$ - the two threads in the same position, $\mathrm{B}$ - the right thread facing up, $\mathrm{C}$ - totally opposite threads and D - , with 3 replicates. The coefficient of variation $(\mathrm{CV})$ fertilizer distribution in relation to the working slope and the position of the threads and amplitude of distribution were as parameters. There was no significant difference between the slopes tested, in level the mean CV was $36.29 \%$, in $-11^{\circ}$ was $37.11 \%$ and $+11^{\circ}$ was $36.57 \%$. Differences occurred in the different work positions and type of threads, with CV at position A of $38.65 \%$, B $37.18 \%$, C $35.63 \%$ and D $35.18 \%$. The dosage shows a relatively high variation of the distribution, but in contrast, this variation does not vary significantly in the factors studied. 


\section{Introdução}

A deposição de fertilizante no solo tem grande importância na obtenção de elevadas produtividades das culturas, estas são dependentes da adubação ao longo de seu cultivo, e os mecanismos dosadores assumem papel determinante neste processo. Os dosadores possuem alguns influenciantes, destes cita-se sua configuração interna, condições de relevo, umidade e tipo de fertilizante.

O ângulo de trabalho do dosador influencia na distribuição de fertilizante, principalmente em terrenos ondulados, como no caso do estado do Rio Grande do Sul - Brasil, assim, o tipo de dosador empregado, afeta a distribuição e a taxa de adubo dosado. Em estudo com diferentes tipos de dosadores de rosca helicoidal, trabalhando em diferentes inclinações longitudinais, [2] as inclinações utilizadas no estudo acarretaram em variação significativa na dosagem de fertilizante granulado. Outro parâmetro que tem influência na distribuição do fertilizante é a espaço que existe no interior do dispositivo dosador, entre a rosca helicoidal e o eixo de transmissão, [1] reduzindo esse espaço há melhoraria na distribuição de fertilizante. A variação na distribuição pode interferir diretamente na produtividade e na rentabilidade do agricultor, pois nos pontos de excesso na linha poderá causar danos à semente ou ao seu sistema radicular, bem como, ao contrário, a falta que não irá suprir adequadamente as necessidades da planta, limitando o rendimento [3].

Um dos principais problemas encontrados em semeadoras é a elevada amplitude de distribuição de fertilizante, com coeficientes de variação podendo chegar até $50 \%$, conforme há alteração da vazão ou da velocidade de trabalho [5]. [8] os problemas enfrentados na semeadura, na maioria das vezes não conseguem ser recuperados no decorrer do ciclo da cultura, influenciando negativamente no seu desempenho final.

No mercado atual do Brasil, os dosadores são compostos com uma rosca helicoidal como mecanismo dosador, nesse ponto, surge um dosador com duas roscas que trabalham em sentidos opostos, buscando segundo fabricante eliminar os pulsos com a rotação contrário entre as mesmas, o que gera dúvidas quanto ao seu desempenho, e nesse sentido esse trabalho teve objetivo de avaliar o desempenho do mecanismo dosador de fertilizantes do tipo rosca helicoidal dupla, em diferentes inclinações de trabalho, diferentes tipos e posições de rosca na dosagem de fertilizante granulado.

\section{Materiais e métodos}

O experimento foi desenvolvido no IFRS - Campus Sertão no Núcleo de Estudos em Solos e Máquinas agrícolas no ano de 2017. Para qualificação do dosador, testes foram realizados em laboratório utilizando-se uma bancada de testes de dosadores de fertilizante (figura 1), equipada com regulagens de inclinação em sentido longitudinal e transversal. Na parte inferior, foi colocado uma esteira acionada por um motor de indução com velocidade constante de 5.4 $\mathrm{km} \cdot \mathrm{h}^{-1}$. O dosador é acionado por um motor elétrico de $1 \mathrm{cv}$ de potência nominal, combinado a um sistema de motoredução controlados por um CLP da marca WEG®. O dosador utilizado para o ensaio foi do tipo rosca helicoidal dupla trabalhando a uma rotação de 54 rpm. Foi empregado

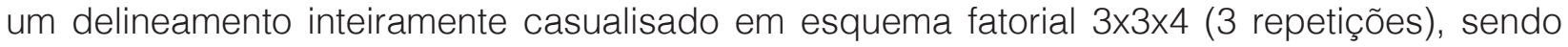
o primeiro fator inclinação de trabalho do dosador, o segundo 3 passos de rosca (figura 2) e 4 posições de acoplamento das roscas no eixo do equipamento (figura 3). As inclinações foram de $+11^{\circ}, 0^{\circ}$ e $-11^{\circ}$, as roscas helicoidais utilizadas foram $22 \mathrm{~mm}, 17 \mathrm{~mm}$, e $34 \mathrm{~mm}$, o posicionamento das roscas foram em todos os sentidos possíveis, sendo que a rosca do lado esquerdo do dosador permaneceu sempre na mesma posição e a segunda foi alterada, sendo a posição A - as duas roscas estavam na mesma posição, B - rosca da direita com o bocal de acoplamento posicionada para cima, C - roscas totalmente contrarias e D - rosca da direita para baixo. O fertilizante utilizado nos testes foi o NPK 2-20-20 com uma dose fixa de $220 \mathrm{~kg} \mathrm{ha}^{-1}$. 

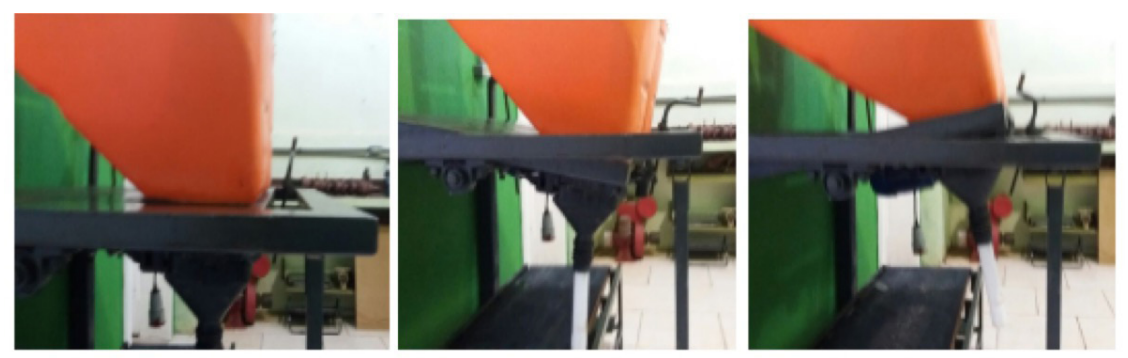

Em nivel 0 은

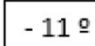

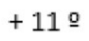

Figura 1. Inclinações de trabalho do dosador de rosca helicoidal dupla nos testes.
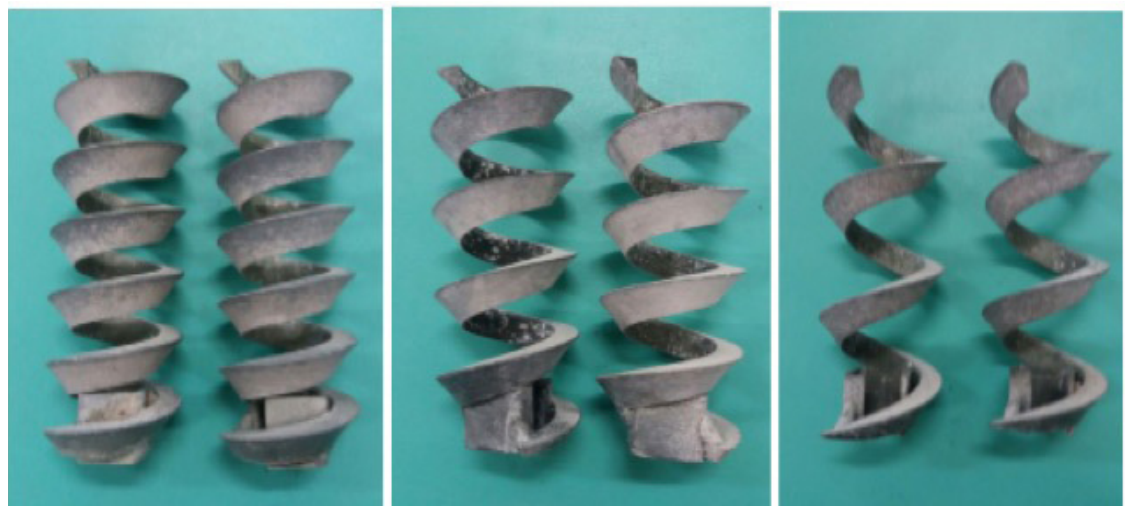

Figura 2. Roscas helicoidais testadas no ensaio.

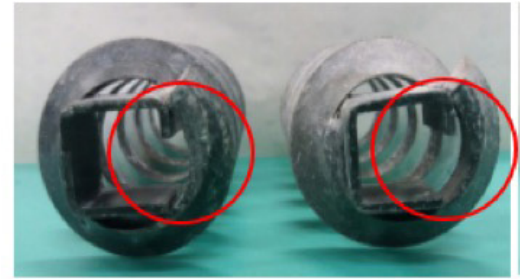

Posição A

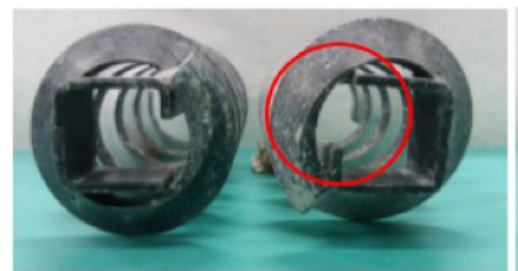

Posição C

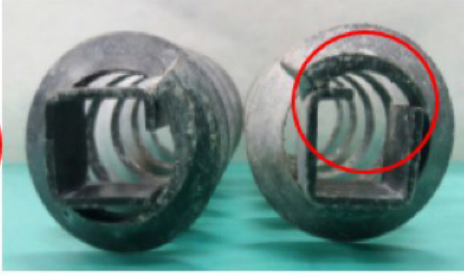

Posição B

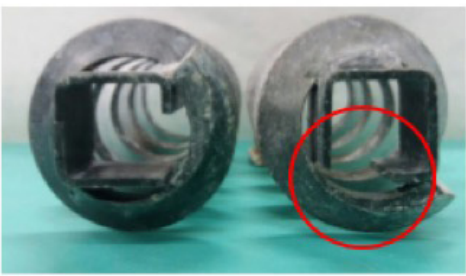

Posição D

Figura 3. Diferentes posições de encaixe da roscas helicoidais duplas ao eixo motor testadas no ensaio.

Para coleta do fertilizante dosado utilizou-se uma calha de metal com as dimensões de 0.09 $x 5.4 \mathrm{~m}$, na qual foram dispostos 60 potes coletores de polietileno com dimensões de 0.09x $0.09 \mathrm{~m}$, um ao lado do outro, sem deixar espaço entre eles. Essa calha coletora foi colocada sob 
a esteira da bancada, sendo que esta era responsável pela movimentação da calha, simulando uma distribuição linear do fertilizante. Onde foi avaliado o coeficiente de variação (CV) da distribuição linear de fertilizante granulado em diferentes condições de trabalho.

Os dados foram tabulados em planilha eletrônica e submetidos à avaliação estatística, que constou de uma análise descritiva, teste de variância, e comparação de médias pelo Teste de Tukey ao nível de 5\% de probabilidade, todas realizadas pelo Assitat 7.7 beta [7].

\section{Resultados e discussão}

Ao observar a tabela 1, notasse que não houve variação significativa no coeficiente de variação da distribuição linear de fertilizante entre as diferentes inclinações de trabalho impostas no dosador de rosca dupla, os valores obtidos ficaram em torno de $36 \%$ de variação, embora seja elevado, o dosador não varia com o relevo, fator que possibilita maior acurácia na qualidade na adubação em locais que possuem relevos acidentados. [2] demonstraram que todas as inclinações proporcionaram variação significativa na dosagem em função da inclinação longitudinal, tanto em dosador de rosca helicoidal por transbordo ou por gravidade, sendo submetidos a inclinações de $10^{\circ}, 5^{\circ}, 0^{\circ},-5^{\circ},-10^{\circ}$.

Em relação ao tipo de roscas empregadas no dosador (tabela 1), a rosca 17mm apresentou a menor variação na distribuição, variando de 31.58 a 35.54\%, sendo que a melhor condição de trabalho foi $0^{\circ}$, que diferiu da rosca $22 \mathrm{~mm}$ e $34 \mathrm{~mm}$ que não diferiram entre si. Esta condição de inclinação não favoreceu a distribuição das roscas $22 \mathrm{~mm}$ e $34 \mathrm{~mm}$, sendo que não houve uma tendência, a primeira teve menor variação quando estava trabalhando em aclive, ou seja, com o trator subindo, com $35.68 \%$ de variação, ja rosca $34 \mathrm{~mm}$ foi na $+11^{\circ}$, contudo não diferiu das demais roscas. Deste modo deduzimos que, o menor passo da rosca, teve a menor variação na distribuição do fertilizante na dosagem utilizada. [4] estudando diferentes dosagens de fertilizante em dosadores de rosca helicoidal, constatou que quanto maior a dose utilizada, menor é o CV na distribuição de fertilizante.

Tabela 1. Coeficientes de variação da dosagem (\%) de fertilizante granulado pelo mecanismo dosador nos diferentes tipos de rosca e inclinações de trabalho.

\begin{tabular}{|c|c|c|c|}
\hline \multirow{2}{*}{ Inclinações } & \multicolumn{2}{|c|}{ Passo da rosca } \\
\cline { 2 - 4 } & $22 \mathrm{~mm}$ & $17 \mathrm{~mm}$ & $34 \mathrm{~mm}$ \\
\hline $0^{\circ}$ & $38.53 \mathrm{aA}$ & $31.58 \mathrm{aB}$ & $38.75 \mathrm{aA}$ \\
\hline$+11^{\circ}$ & $39.52 \mathrm{aA}$ & $35.54 \mathrm{aA}$ & $36.28 \mathrm{aA}$ \\
\hline$-11^{\circ}$ & $35.68 \mathrm{aB}$ & $33.44 \mathrm{aB}$ & $40.1 \mathrm{aA}$ \\
\hline
\end{tabular}

*Médias seguidas pela mesma letra maiúscula na linha, ou minúscula na coluna não diferiram estatisticamente entre si, pelo teste de Tukey ao nível de $5 \%$ de probabilidade.

Na combinação posição da rosca e inclinação (tabela 2), as menores variações na distribuição foram obtidas quando o dosador estava com a posição de rosca $\mathrm{C}$ e em declive, obtendo uma 
variação de 31.84\% e também quando o dosador estava em nível $\left(0^{\circ}\right)$ com as roscas na posição D, com variação de 33.35\%, no restante das situações a variação ficou em torno de 37\%.

Quando observamos a posição das roscas, percebemos que as posições A e B não diferiram significativamente nos ângulos $0^{\circ} \mathrm{e}+11^{\circ}$, diferença houve apenas nos $-11^{\circ}$, em que a posição C, menor coeficiente de variação diferiu de todas posições exceto a D. Trabalhos com alteração da posição não há na bibliografia, bem como, testando esse dosador que está há um ano somente no mercado.

Tabela 2. Coeficientes de variação de dosagem (\%) de fertilizante granulado pelo mecanismo dosador na interação posição de encaixe da rosca com inclinação de trabalho.

\begin{tabular}{|c|c|c|c|c|}
\hline \multirow{2}{*}{ Inclinações } & \multicolumn{3}{|c|}{ Posição da rosca } \\
\cline { 2 - 5 } & $\mathrm{A}$ & $\mathrm{B}$ & $\mathrm{C}$ & $\mathrm{D}$ \\
\hline $0^{\circ}$ & $38.80 \mathrm{aA}$ & $36.51 \mathrm{abA}$ & $36.51 \mathrm{abA}$ & $33.35 \mathrm{aAB}$ \\
\hline$+11^{\circ}$ & $38.18 \mathrm{aA}$ & $34.43 \mathrm{bA}$ & $38.55 \mathrm{aA}$ & $37.30 \mathrm{aA}$ \\
\hline$-11^{\circ}$ & $38.98 \mathrm{aA}$ & $40.59 \mathrm{aA}$ & $31.84 \mathrm{bB}$ & $34.89 \mathrm{aA}$ \\
\hline
\end{tabular}

*Médias seguidas pela mesma letra maiúscula na linha, ou minúscula na coluna não diferiram estatisticamente entre si, pelo teste de Tukey ao nível de $5 \%$ de probabilidade.

Na interação passo da rosca com posição de encaixe da mesma (tabela 3) novamente o menor passo $(17 \mathrm{~mm})$ apresentou os menores variações dos tipos de roscas, sendo que e melhor posição foi a posição D para essa rosca, com 32.23\% que não diferiu entre as posições de encaixe, mostrando que nesse tipo de rosca tanto faz a forma de montagem da mesma. Tal fato deve estar ligado que no menor passo os pulsos são reduzidos.

Aqui é visível que o maior passo da rosca, maiores são as variações, confirmando que independentemente da posição da inclinação, menores passos apresentam as maiores variações.

Tabela 3. Coeficientes de variação de dosagem (\%) de fertilizante granulado pelo mecanismo dosador na interação da posição de encaixe da rosca com o tipo de passo rosca.

\begin{tabular}{|c|c|c|c|c|}
\hline \multirow{2}{*}{ Passo da rosca } & \multicolumn{4}{|c|}{ Posição da rosca } \\
\cline { 2 - 5 } & A & B & C & D \\
\hline $22 \mathrm{~mm}$ & $37.16 \mathrm{bAB}$ & $38.75 \mathrm{aAB}$ & $41.05 \mathrm{aA}$ & $34.68 \mathrm{abB}$ \\
\hline $17 \mathrm{~mm}$ & $33.51 \mathrm{bA}$ & $36.05 \mathrm{aA}$ & $32.31 \mathrm{bA}$ & $32.23 \mathrm{bA}$ \\
\hline $34 \mathrm{~mm}$ & $45.29 \mathrm{aA}$ & $36.74 \mathrm{aB}$ & $33.52 \mathrm{bB}$ & $38.62 \mathrm{aB}$ \\
\hline
\end{tabular}

*Médias seguidas pela mesma letra maiúscula na linha, ou minúscula na coluna não diferiram estatisticamente entre si, pelo teste de Tukey ao nível de $5 \%$ de probabilidade. 


\section{Conclusões}

O dosador de rosca helicoidal dupla apresenta coeficiente de variação da distribuição linear de fertilizante granulado média de $36 \%$, este não tem variação em relação as inclinações de trabalho. Quanto menor o passo da rosca menor é a variação da distribuição, sendo que a posição de encaixe da rosca não gera alterações significativas no seu desempenho.

\section{Referencias}

[1] G. J. Bonotto. Desempenho de dosadores de fertilizantes de semeadoras-adubadoras em linhas. Tese de Doutorado. Centro de Ciências Rurais - Universidade Federal de Santa Maria, Santa Maria, UFSM. Rio Grande do Sul, 2012.

[2] M.F.P Ferreira, et al. Uniformidade de vazão de fertilizantes por dosadores helicoidais em função do nivelamento longitudinal. Engenharia na agricultura., vol. 18, no. 4, pp. 297-304, 2010.

[3] L. Pagnussat, et al. Pulso e eficiência de dosadores de rosca sem fim em função da dosagem. In: Congresso Brasileiro de Engenharia Agrícola, n 43, Fortaleza/CE.2013.

[4] L. Pagnussat, et al. Eficiência de dosadores helicoidais em função da dosagem na cultura da soja. In: Congresso Brasileiro de Engenharia Agrícola, no. 44, Campo Grande/MS.2014.

[5] J.A. Portella; A. Sattler; A. Faganello. Regularidade da distribuição de sementes e de fertilizantes em semeadoras para plantio direto de trigo e soja. Engenharia Agrícola., vol. 17, no. 4, pp.57-64, 1998.

[6] A. Sattler; J. A. Portella; A. Faganello. Estudo preliminar da vazão de um fertilizante sólido em semeadoras-adubadoras. In: Congresso Brasileiro de Engenharia Agrícola, no. 31, Pelotas/RS. 1999.

[7] F.A.S.E. Silva; C. A. V. Azevedo. Principal Components Analysis in the Software Assistat-Statistical Attendance. In: World Congress on Computers in Agriculture, no. 7, Reno-NV-USA. 2009

[8] A.V. dos. Reis. Erros na semeadura. Cultivar Máquinas., vol. 1, no. 2, pp. 12-13, 2001. 